\title{
OUTCOME OF BONE TUBERCULOSIS IN CHILDREN IN RURAL INDIA - A CASE SERIES
}

\author{
Aarti Rahangdale. \\ Consultant Pediatrician, Aadhya Multispecialty Hospital, Gondia, Maharashtra, India.
}

\begin{abstract}
Bone tuberculosis (TB) is relatively uncommon form of extrapulmonary TB in children. It is estimated that 1-6 \% children with primary infection may develop bone and joint TB in 1-3 years if left untreated. Though short course chemotherapy has been found to be effective in treating bone and joint $T B$, due to emergence of multi drug resistant (MDR) TB, the incidence of resistant bone TB is also rising. Here we have 5 cases of bone TB affecting different sites and having a broad spectrum of antitubercular drug sensitivity pattern ranging from drug sensitive bone TB to MDR bone TB.
\end{abstract}

\author{
ARTICLE HISTORY \\ Received 3 June 2021 \\ Accepted 16 July 2021
}

\section{KEYWORDS}

\section{Introduction}

About 2.2 million new cases of tuberculosis (TB) occur every year. As per the World Health Organization (WHO) 1999 report on Global TB control, Southeast Asia accounts for approximately $40 \%$ cases in the world and more than $95 \%$ of cases are found in India, Indonesia, Bangladesh, Thailand and Myanmar. ${ }^{1}$ In India, $85 \%$ of them have pulmonary TB and $15 \%$ have extrapulmonary lesions of which $1-3 \%$ are bone and joint TB. ${ }^{2}$

TB of bone and joints is rampant in India with the dorsolumbar spine as the most common site of osseous involvement. ${ }^{3,4}$ For diagnosis, clinical suspicion needs to be confirmed through appropriate laboratory and imaging investigations and increasingly nowadays nucleic acid amplification technique. Though short course chemotherapy has been found to be effective in treating bone and joint TB, due to emergence of multi-drug resistant (MDR) pulmonary TB (PTB), the incidence of resistant bone TB is also rising. ${ }^{1}$ We present 5 cases with bone TB of which one had drug resistant TB.

Case 1:- A 6-year-old boy presented with swelling on right side of chest for which he was seen by an orthopedic surgeon and Chest X-Ray was done that was suggestive of osteomyelitis of the $7^{\text {th }} \& 9^{\text {th }}$ rib. Ultrasound of chest showed heterogenous swelling over right side of chest wall measuring $3.3 \times 1.8 \times 1.4 \mathrm{~cm}$ with erosion of $7^{\text {th }} \& 9^{\text {th }}$ rib. Surgical debridement was done and was suggestive of cold abscess. Pus culture grew mycobacterium tuberculosis (MTB) which was sensitive to all the antituberculous drugs. Child was started on antituberculous therapy (ATT) as depicted in Table 1. There was no contact with a patient of TB. He had double outlet Right Ventricle (DORV) for which he was operated at 18 months of age. On examination,

Address for Correspondance: Aadhya Multispecialty Hospital, Ring Road, Rajabhoj Colony, Gondia 661614, Maharashtra, India.

Email: dr.aarti.r@gmail.com

@2021 Pediatric Oncall weight was $16 \mathrm{~kg}$ and length were $110 \mathrm{~cm}$. Systemic examination was normal. He gained $2 \mathrm{~kg}$ during treatment and is now asymptomatic.

Case 2:- A 4-year-old girl presented with pain in right hip for 4 months. There was no fever or contact with a patient of TB. There was no loss of weight or loss of appetite. X-Ray of pelvis was done that showed lytic lesion in right femoral head with irregularity. Her Chest X-Ray, Mantoux test was negative. She was started on ATT as depicted in Table 1 . On presentation, her weight was $12 \mathrm{~kg}$, height was $91 \mathrm{cms}$. Systemic examination was normal. Her ATT was continued and after completion of 9 months of ATT, MRI lumbar spine showed partial collapse of L5 vertebra with altered marrow signal at right femoral head. She was advised continuation of ATT for a longer time but was subsequently lost to follow up.

Case 3:- A 6-year-old boy presented with pain and swelling in right leg 6 months ago and was diagnosed as closed fracture of right tibial shaft. His leg was put in plaster for 2 months. Subsequently he was alright and then again developed pain and swelling at the same area 4 months later. There was no fever or TB contact. An X-ray of the right lower limb showed lytic lesion in the shaft of the tibia. A surgical debridement was done, and it showed presence of acid-fast bacillus (AFB) on smear. Child was started ATT schedule as shown in Table 1. On examination he had puckered sear over right tibia, height was $115 \mathrm{~cm}$, weight was $19 \mathrm{~kg}$. Other systems were normal. He was continued on ATT and after 9 months of ATT, had put on $2 \mathrm{~kg}$ weight and $X$-Ray of the leg was normal.

Case 4:- An 11-year-old girl presented with pain in lower back and gait abnormality. She was diagnosed as TB spine and started on ATT which she took only for 3 months as she had relief of symptoms. Again, the child started getting back pain with shooting pain in both lower limbs and a limp on walking. She had cough with expectoration. There was no fever or contact with a patient of TB. On examination, height was 120 $\mathrm{cms}$, weight was $20 \mathrm{~kg}$. She had insignificant bilateral cervical lymph nodes. There was gibbus over $\mathrm{T}_{12}-$ 
$\mathrm{L}_{2}$ vertebrae with tenderness over dorsal and lumbar spine and antalgic gait. Central nervous system (CNS) system examination was normal. Other systems were normal. X-Ray spine showed TB spine with collapse of $T_{12}, L_{3}$ and $L_{4}$ vertebra. She was advised MRI spine and Mantoux test but was lost to follow up.

Case 5:- A 7-year-old male presented for management of his TB. Three years ago, he had fever with evening rise of temperature along with loss of weight for $2 \frac{1}{2}$ months. Chest X-Ray showed right sided upper zone collapse. He was treated with $1^{\text {st }}$ line ATT for 6 months. He was then asymptomatic. Currently, the child again had fever for 1 month along with right elbow swelling and cervical adenopathy. Cervical lymph node biopsy showed AFB on smear and both ulnar biopsy and cervical lymph node biopsy were positive for TB PCR. Culture showed resistance to isoniazid $(H)$, rifampicin $(R)$, pyrazinamide $(Z)$ and ethambutol (E). He was started on injectable kanamycin which he received for 3 months, ofloxacin, cycloserine, clofazimine \& ethionamide which were given for 2 years. At the end of 2 years, all findings were normal. The cervical glands had regressed, and elbow swelling had subsided. Child was thus stopped on ATT.

\section{Discussion}

In India, the incidence of osteoarticular TB appears to be higher in children, adolescents and young adults, than in elderly because epiphysial region of bone is highly vascular in infant and young children. ${ }^{1}$ The spine is probably the most common site of osseous involvement followed by hip and knee. ${ }^{1}$ Spinal TB is nearly equal to TB of all the bony region put together. In our patients, only one child had spinal TB. Bone and joints TB is almost always secondary to a primary focus in the lymphatic glands or lungs or mesentery from where it disseminates by hematogenous route. ${ }^{2}$ Also associated malnutrition or HIV infection are more prone to develop TB. ${ }^{3,5}$ None of our patients had HIV though one did have drug resistant TB. The human bacillus is responsible for almost all the case of osteoarticular TB in India. ${ }^{6}$ Cold abscesses are the most common and important criteria for establishing diagnosis of TB of the spine. Hence anatomical path of the cold abscess is of great important because the anatomical presentation of the cold abscess can be far away from the site of infection. ${ }^{3}$ In children the lesion is commonly of the caseating type with rapid and extensive distribution of bone and cartilage whereas in adult the proliferating type with less bone destruction in usual. ${ }^{7}$ The initial involvement in bone is usually the epiphyses or metaphysis. The tubercular bony lesion in children is less sclerotic as compared to adult. ${ }^{7}$

Involvement of hip joint is second commonest skeletal lesion next to that of spine. ${ }^{1}$ In late stages, when destruction has been progressive, the limb goes into flexion, abduction and internal rotation with an apparent limb shortening. ${ }^{3}$ In ankle joint, the most common site of lesions are tibia, fibula and talus. Tuberculous osteomyelitis occurs in about $1-6 \%$ of patients with bone and joint TB. ${ }^{7}$ In most of them the skeletal site of lesion is multiple like manubrium sterni, sternum and isolated spinous processes. Our remaining patients had femoral osteomyelitis, tibial osteomyelitis and ulnar osteomyelitis and rib involvement.

The onset of TB in any bone or joint is insidious. Constitutional symptoms like low grade fever, anorexia and weight loss usually proceeds localizing symptoms and signs such as pain, tenderness and swelling of affected part. ${ }^{5}$ If the spine is involved truncal rigidity, muscle spasms and neurological signs may be present. ${ }^{1}$ CT scans helps to define the extent of soft tissue calcification and will help to distinguish spinal TB from other condition. ${ }^{5}$ MRI is practically helpful in spinal TB to show the severity and content of extradural compression and helps to differentiate between an abscess and fibrous tissue. Bacteriological confirmation by identification of the bacillus in cold abscess aspirate or biopsy taken from the site of the lesion (bone shaft or granulation tissue) by CBNAAT or culture of bacilli would be necessary in certain cases. ${ }^{8}$ In Indian scenario, various studies have shown varying rate of confirmation : $40-80 \%$ by Dahl, $60.5 \%$ by Tuli, and $87 \%$ by Lakhanpal after culture and guinea pig inoculation. ${ }^{8}$

Table 1. Clinical profile of all patients

\begin{tabular}{|c|c|c|c|c|c|}
\hline & Patient 1 & Patient 2 & Patient 3 & Patient 4 & Patient 5 \\
\hline Age & 6 years & 4 years & 6 years & 11 years & 7 years \\
\hline Gender & Male & Female & Male & Female & Male \\
\hline Clinical features & $\begin{array}{l}\text { Rib } \\
\text { osteomyelitis }\end{array}$ & $\begin{array}{l}\text { Femoral } \\
\text { osteomyelitis }\end{array}$ & $\begin{array}{l}\text { Tibial } \\
\text { osteomyelitis }\end{array}$ & Pott's spine & $\begin{array}{l}\text { TB Lymphadenopathy } \\
+ \text { ulnar osteomyelitis }\end{array}$ \\
\hline Drug sensitivity & $\begin{array}{l}\text { Sensitive all } \\
\text { Anti TB drugs }\end{array}$ & Not done & Not done & Not done & Resistant $=\mathrm{HRZE}$ \\
\hline ATT regime & $\begin{array}{l}2 \mathrm{HRZE}+4 \\
\mathrm{HRE}\end{array}$ & $\begin{array}{l}2 \mathrm{HRZE}+7 \\
\mathrm{HRE}\end{array}$ & $\begin{array}{l}2 \mathrm{HRZE}+7 \\
\mathrm{HRE}\end{array}$ & Not started & $\begin{array}{l}\text { Kanamycin ( } 3 \text { mths) } \\
\text { Ofloxacin ( } 2 \text { yrs) } \\
\text { Ethionamide ( } 2 \text { yrs) } \\
\text { Cycloserine ( } 2 \text { yrs) } \\
\text { Clofazimine ( } 2 \text { yrs) }\end{array}$ \\
\hline Follow-up & Recovered & Same & Recovered & $\begin{array}{l}\text { Lost to follow } \\
\text { up }\end{array}$ & Recovered \\
\hline
\end{tabular}

Note: $\mathrm{H}=$ Isoniazid, $\mathrm{R}=$ Rifampicin, $\mathrm{Z}=$ Pyrazinamide, $\mathrm{E}=$ Ethambutol, $\mathrm{S}=$ Streptomycin 
The treatment of skeletal TB is medical, however open surgical biopsy is often necessary and large abscesses need to be drained. The indication for surgery is neurological involvement, spinal instability, failure of medical treatment and significant kyphotic deformity. ${ }^{3,5}$ In osteoarticular TB in children the regenerative ability of the epiphyses and the articular cartilage is very high and antitubercular drugs gives excellent result. ${ }^{9}$ In Madras study in collaboration with TB Research center of Indian Council of medical research, ambulatory treatment with rifampicin and isoniazid for 9 months was found to be superior in $99 \%$ of patient with 10 years of follow up. ${ }^{1}$ Therefore uncomplicated TB of the spine is a medical disease. In a clinical practice, if the disease is not showing a healing response within 4-5 months despite multidrug therapy, one should consider such cases as "resistant" and treated accordingly. ${ }^{10}$ One of our patients had drug resistant TB and she responded very well to the second line drugs.

\section{Conclusion}

Bone TB lesions may present in a bizarre fashion (as in our cases) and may mimic other similar bony lesions. Early diagnosis and treatment of bone TB is again essential to stop further hematogenous spread of TB to other bones. Hence a high index of suspicion and timely treatment of apparently solitary skeletal lesion may reduce the incidence of multifocal cystic TB in children.

\section{Compliance with Ethical Standards}

Funding None

Conflict of Interest None

\section{References:}

1. Hazra A, Laha B. Chemotherapy of osteoarticular tuberculosis. Indian J Pharmacol. 2005; 37: 5-12.

2. Shanmugasundaram TK. Bone and joint tuberculosis -Guidelines for management. Indian J Orthop, 2005; 39: 195-198.

3. Seth V, Kabra SK, Lodha R. Essentials of Tuberculosis in Children. 3rd Edition, 2006: 548-549.

4. Tuli SM, Srivastava TP, Varma BP, Sinha GP. Tuberculosis of spine. Acta Orthop Scand. 1967;38: 445-458.

5. Mousa H AL. Bones and Joints Tuberculosis Bahrain Medical Bulletin. 2007;29:1-9. Available at URL: https://www. bahrainmedicalbulletin.com/ march_2007/Bones_Joints_ Tuberculosis.pdf. Accessed on 5th May 2021.

6. Vyaghreswarudu $C$. Bone tuberculosis in children. Indian J Pediatri. 1952:20:162-167.

7. Malik S, Joshi S, Tank JS. Cystic bone tuberculosis in children--a case series. Indian J Tuberc. 2009;56:220-224.

8. Sankaran B. Tuberculosis of Bones and Joints. Indian J Tuber. 1993;40,109.

9. Papavasiliou VA, Petropoulos AV. Bone and Joint TB in Childhood. Acta Orthop Scand. 1981;52,1-4.

10. Tuli SM. Challenge of therapeutically refractory and multidrug resistant tuberculosis in orthopaedic practice. Indian J Orthop. 2002;36:211-213. 